\title{
Nivolumab induced hypophysitis in a patient with recurrent non-small cell lung cancer
}

\author{
Smile Kajal $^{1}$, Pooja Gupta ${ }^{2, *}$, Anam Ahmed ${ }^{3}$, Anurag Gupta ${ }^{4}$ \\ ${ }^{1}$ Department of Otolaryngology, All India Institute of Medical Sciences (AIIMS), New Delhi, India; \\ ${ }^{2}$ Department of Pharmacology, All India Institute of Medical Sciences (AIIMS), New Delhi, India; \\ ${ }^{3}$ Department of Anatomy, All India Institute of Medical Sciences (AIIMS), New Delhi, India; \\ ${ }^{4}$ Department of Pathology, University College of Medical Sciences, New Delhi, India.
}

SUMMARY Nivolumab is a programmed death receptor-1 blocking monoclonal antibody which has been approved by United States Food and Drug Administration for patients with metastatic non-squamous non-small cell lung cancer. Endocrinopathies like thyroid dysfunction and adrenal insufficiency are its known immune related adverse effects. Hypophysitis is very rare and usually presents with minimal symptoms. We report development of hypophysitis in an 84-year-old female patient who developed a range of symptoms (fatigue, headache, nausea) as well as laboratory confirmation of both central hypothyroidism and central adrenal deficiency which is unusual in cases of nivolumab induced hypophysitis. The patient had well differentiated adenocarcinoma of the left upper lobe of the lung. She underwent wedge resection followed by chemotherapy and was started on nivolumab due to recurrence. After 14 cycles of nivolumab, she started complaining of intense fatigue. She was found to have central thyroid deficiency and was started on levothyroxine. But her symptoms did not improve. Then she underwent adrenocorticotropic hormone stimulation test which showed central adrenal deficiency, but her brain magnetic resonance imaging did not reveal any pituitary or sellar changes. A diagnosis of nivolumab induced hypophysitis was made, based on clinical grounds and hormonal profile and she was started on oral steroids. She responded dramatically to this steroidal therapy within four weeks of its initiation and her immunotherapy with nivolumab was restarted.

Keywords Nivolumab, hypophysitis, non-small cell lung cancer, hypopituitarism

\section{Introduction}

Nivolumab is a monoclonal antibody of immunoglobulin G4 type which targets the programmed death-1 (PD1) receptor. It was approved by United States Food and Drug Administration (FDA) in October 2015 for patients with metastatic non-squamous non-small cell lung cancer (NSCLC) based on the CheckMate 057 trial that showed improvement in overall survival as compared to docetaxel in patients who progressed on or after platinum-based chemotherapy. It has also been approved for the treatment of advanced melanoma, malignant pleural mesothelioma, advanced renal cell carcinoma, classical Hodgkin's lymphoma, advanced squamous cell carcinoma of head and neck, urothelial carcinoma, metastatic colorectal cancer, hepatocellular carcinoma, and advanced esophageal squamous cell carcinoma $(1,2)$. The main immune-related adverse events (irAEs) associated with nivolumab include diarrhea, colitis, hepatitis, skin toxicities and endocrinopathies such as hypophysitis and thyroid dysfunction (3). Although thyroid dysfunction is a common adverse effect of nivolumab, hypophysitis is rare with an incidence of less than $1 \%$ and only a handful cases of nivolumab induced hypophysitis in patients with NSCLC have been described in literature (4-6). Usually, such cases present with less symptoms and radiological features as compared to other immunotherapy related hypophysitis $(5,6)$. We present a novel case of an elderly patient with recurrent NSCLC who was diagnosed with nivolumab induced hypophysitis based on clinical suspicion due to her hormonal profile and a range of symptoms which she developed during nivolumab immunotherapy.

\section{Case Report}

An 84-year-old Caucasian female, who was first diagnosed with well differentiated adenocarcinoma of the left upper lobe of lung in 2009, underwent left 
Table 1. Result of Adrenocorticotropic hormone (ACTH) stimulation test done in patient

\begin{tabular}{llccc}
\hline Hormone & Reference Range & $\begin{array}{c}\text { Baseline levels } \\
(10: 15 \mathrm{am})\end{array}$ & $\begin{array}{c}30 \text { mins after ACTH } \\
\text { stimulation }\end{array}$ & $\begin{array}{c}60 \mathrm{mins} \text { after ACTH } \\
\text { stimulation }\end{array}$ \\
\hline ACTH $(\mathrm{pg} / \mathrm{mL})$ & $10-48$ & 8 & - & 24.7 \\
Cortisol $(\mathrm{mcg} / \mathrm{dL})$ & $6.0-18.4(7-10 \mathrm{am})$ & 9.7 & & 29.8 \\
Aldosterone $(\mathrm{ng} / \mathrm{dL})$ & $2.7-10.5(3-6 \mathrm{pm})$ & $<1.0$ & 4.8 \\
\hline
\end{tabular}

upper lobe wedge resection and mesh brachytherapy in November 2009. She did not have history of smoking and had hypertension controlled with oral medications. She was put on systemic chemotherapy with pemetrexed, and carboplatin and the treatment response was monitored with Computed Tomography (CT) imaging of chest, abdomen, and pelvis. In January 2012, her CT scan images showed disease recurrence at the wedge resection site, thereby increasing the size of the right upper lobe, and left upper lobe nodules. The tumor was EGFR (epidermal growth factor receptor) and EML 4/ALK (echinoderm microtubuleassociated protein-like 4/anaplastic lymphoma kinase) mutation negative. She then completed her systemic chemotherapy with pemetrexed and carboplatin in March 2012 which was followed by oral erlotinib for four years. Erlotinib had to be discontinued in January 2016 due to continued adverse effects. Later, in March 2016 she was started on nivolumab immunotherapy which was well tolerated till October 2016 (11 cycles) when she complained of intense fatigue occasionally which led to prolonged sleeping. Occasional headaches were also an added complaint accompanied with dryness of mouth and eyes. Her thyroid profile showed low thyroid stimulating hormone (TSH) and low thyroid hormone (T4). However, no thyroid autoantibodies were found in blood, and she was started on oral thyroxine. By November 2016 she had completed 14 cycles of nivolumab immunotherapy and still had similar complaints of fatigue, increased sleepiness, and headaches during her office visits in December 2016. The patient received one more treatment with nivolumab in mid-December 2016 post which a 2-month break from the treatment was approved. On continuing with the same treatment in February 2017, she complained of frequent episodes of lightheadedness and nausea. Her fatigue and headache had still not improved but she did not have any visual symptoms. Adrenocorticotropic hormone (ACTH) stimulation test confirmed central adrenal deficiency (Table 1). Her brain magnetic resonance imaging (MRI) did not show any significant abnormality. A diagnosis of depressed anterior pituitary function was made owing to her earlier decreased TSH and now central adrenal deficiency. The patient was started on oral glucocorticoids (Tablet prednisolone 1 $\mathrm{mg} / \mathrm{kg}$ for a week and then tapering dose over a month). The patient's symptoms subsided within four weeks. Nivolumab immunotherapy was restarted and the low dose prednisolone along with same dose levothyroxine was continued till the end of therapy.

\section{Discussion}

Hypophysitis is an acute or chronic inflammation of pituitary gland. It is a rare condition in the general population and its incidence has not yet been quantified. However, less than $1 \%$ of surgically treated pituitary lesions demonstrate histological features of hypophysitis (7). Autoimmune hypophysitis, which is a subtype, has a prevalence of about 5 per million and an annual incidence of 1 in 7 to 9 million (8). Immunotherapyassociated hypophysitis is commonly associated with headache and anterior hypopituitarism. Anterior hypopituitarism presents with a characteristic but atypical pattern of deficiency of ACTH followed by TSH, gonadotrophins and prolactin deficiency or hyperprolactinemia (9). The degree of pituitary enlargement is generally mild which goes undetected on MRI or is completely absent, and compression of the optic apparatus is extremely rare $(5,6,9)$.

Prior to the nivolumab, hypophysitis was relatively common in patients treated with ipilimumab, a human monoclonal antibody against the cytotoxic T-Lymphocyte antigen 4 (CTLA-4 Ab), which was approved by the FDA in 2011 for the treatment of advanced melanoma (10-15). It has been seen that as compared to ipilimumab associated hypophysitis, nivolumab induced hypophysitis is still rare, is diagnosed late (median of 25 weeks) and is less commonly associated with any symptoms like headache or any pituitary changes detected on MRI $(5,6)$. However, our patient presented with a range of symptoms (fatigue, headache, nausea) as well as laboratory confirmation of both central hypothyroidism and central adrenal deficiency which is unusual in cases of nivolumab induced hypophysitis. A review of the trial data of immune checkpoint-induced hypophysitis showed that the incidence of hypophysitis for ipilimumab was $0-17 \%$ $(6,16,17)$ while the incidence was less for nivolumab $(<$ $1 \%)(7,18)$ and pembrolizumab $(<1 \%)(7,19)$.

In a retrospective analysis of 83 patients treated with immunotherapy to observe the immune checkpoint inhibitor related hypophysitis (irH), the irH was defined as (i) ACTH or TSH deficiency plus MRI changes or (ii) ACTH and TSH deficiencies plus headache/ fatigue in the absence of MRI findings. As per this 
definition, 62 patients had irH at initial evaluation and the most common symptom was fatigue $(66 \%)$ followed by headache $(60 \%)$. Central hypothyroidism and central adrenal insufficiency were seen in $94 \%$ and $69 \%$ patients, respectively and $77 \%$ of the $\mathrm{irH}$ patients had MRI changes like stalk thickening, suprasellar convexity, and heterogeneous enhancement of the pituitary gland. 48 out of $62(77 \%)$ patients were on ipilimumab, 2/62 (3\%) on tremelimumab, 3/62 (5\%) on nivolumab, and 9/62 (15\%) on a combination of both nivolumab and ipilimumab. When compared to therapies without ipilimumab, those with ipilimumab had a statistically significant association with $\operatorname{irH}(\mathrm{p}<$ 0.01 ) and the median time interval from initiation of immunotherapy to development of irH was less for ipilimumab (9-12 weeks) as compared to nivolumab (30.4-37.7 weeks) (20).

Patients with irH have been treated with physiologic to high-dose glucocorticoids resulting in improvement of symptoms and pituitary function. In most patients, the steroids and other hormone replacements, like for thyroid, need to be continued till the end of immunotherapy or even after the end of immunotherapy as the recovery rate is variable. The chance of recovery of thyroidal axis is more than the adrenal or gonadal axis $(5,6,9,20)$.

Our patient developed central hypothyroidism followed by central adrenal deficiency as evident by ACTH stimulation test. Her brain MRI did not show any significant finding, but she had history of episodes of headache, prolonged sleepiness, and nausea. Her presentation was novel for nivolumab induced hypophysitis and a diagnosis was made, based on clinical grounds and hormonal profile. She was started on glucocorticoids to which she responded well symptomatically which further supported our diagnosis. In almost all cases of immune mediated hypophysitis, the histopathological diagnosis of hypophysitis by biopsy of pituitary gland is never indicated due to increased risk-to-benefit ratio. Hence, a high degree of clinical suspicion is required for its timely diagnosis.

\section{Conclusion}

Immunotherapy induced hypophysitis is a rare condition and has frequently been described with Anti-CTLA4 agents like ipilimumab, but it is rare with Anti-PD1 agents like nivolumab and usually presents with less symptoms. A normal MRI of the brain does not exclude hypophysitis. Therefore, a high degree of clinical suspicion and multidisciplinary team involving medical oncologists, clinical pharmacologists, endocrinologists, and radiologists is required to diagnose such cases.

\section{Acknowledgements}

We would like to thank Dr Moses Raj (Associate
Professor, Temple University, Allegheny Health Network Cancer Institute, Pittsburgh, United States) for his expert opinion on this case.

\section{Funding: None.}

Conflict of Interest: The authors have no conflicts of interest to disclose.

\section{References}

1. Keating GM. Nivolumab: A review in advanced non squamous non-small cell lung cancer. Drugs. 2016; 76:969-978.

2. Kazandjian D, Suzman DL, Blumenthal G, Mushti S, He K, Libeg M, Keegan P, Pazdur R. FDA approval summary: Nivolumab for the treatment of metastatic non-small cell lung cancer with progression on or after platinum-based chemotherapy. Oncologist. 2016; 21:634-642.

3. Spain L, Diem S, Larkin J. Management of toxicities of immune checkpoint inhibitors. Cancer Treat Rev. 2016; 44:51-60.

4. Ishikawa M, Oashi K. Case of hypophysitis caused by nivolumab. J Dermatol. 2017; 44:109-110.

5. Garon-Czmil J, Petitpain N, Rouby F, Sassier M, Babai $\mathrm{S}$, Yéléhé-Okouma M, Weryha G, Klein M, Gillet P. Immune check point inhibitors-induced hypophysitis: a retrospective analysis of the French pharmacovigilance database. Sci Rep. 2019; 9:19419.

6. Faje A, Reynolds K, Zubiri L, Lawrence D, Cohen JV, Sullivan RJ, Nachtigall L, Tritos N. Hypophysitis secondary to nivolumab and pembrolizumab is a clinical entity distinct from ipilimumab-associated hypophysitis. Eur J Endocrinol. 2019; 181:211-219.

7. Joshi MN, Whitelaw BC, Palomar MT, Wu Y, Carroll PV. Immune checkpoint inhibitor-related hypophysitis and endocrine dysfunction: Clinical review. Clin Endocrinol. 2016; 85:331-339.

8. Howlett TA, Levy MJ, Robertson IJ. How reliably can autoimmune hypophysitis be diagnosed without pituitary biopsy. Clin Endocrinol (Oxf). 2010; 73:18-21.

9. Faje A. Hypophysitis: Evaluation and management. Clin Diabetes Endocrinol. 2016; 2:15.

10. Faje A. Immunotherapy and hypophysitis: clinical presentation, treatment, and biologic insights. Pituitary. 2016; 19:82-92.

11. Faje AT, Sullivan R, Lawrence D, Tritos NA, Fadden R, Klibanski A, Nachtigall L. Ipilimumab induced hypophysitis: a detailed longitudinal analysis in a large cohort of patients with metastatic melanoma. J Clin Endocrinol Metab. 2014; 99:4078-4085.

12. Chodakiewitz Y, Brown S, Boxerman JL, Brody JM, Rogg JM. Ipilimumab treatment associated pituitary hypophysitis: clinical presentation and imaging diagnosis. Clin Neurol Neurosurg. 2014; 125:125-130.

13. Lam T, Chan MM, Sweeting AN, De Sousa SM, Clements A, Carlino MS, Long GV, Tonks K, Chua E, Kefford RF, Chipps DR. Ipilimumab-induced hypophysitis in melanoma patients: an Australian case series. Intern Med J. 2015; 45:1066-1073.

14. Ryder M, Callahan M, Postow MA, Wolchok J, Fagin JA. Endocrine-related adverse events following 
ipilimumab in patients with advanced melanoma: a comprehensive retrospective review from a single institution. Endocr Relat Cancer. 2014; 21:371-381.

15. Gambale E, Tinari C, Carella C, Paolo AD, Tursi MD. Immunotherapy and hypophysitis in the clinical practice: A case report. J Clin Case Rep. 2016; 6:861.

16. Corsello SM, Barnabei A, Marchetti P, De Vecchis L, Salvatori R, Torino F. Endocrine side effects induced by immune checkpoint inhibitors. J Clin Endocrinol Metabol. 2013; 98:1361-1375.

17. Torino F, Barnabei A, Paragliola RM, Marchetti P, Salvatori R, Corsello SM. Endocrine side-effects of anti-cancer drugs: mAbs and pituitary dysfunction: clinical evidence and pathogenic hypotheses. European J Endocrinol. 2013; 169:R153-R164.

18. Topalian SL, Sznol M, McDermott DF, et al. Survival, durable tumor remission, and long-term safety in patients with advanced melanoma receiving nivolumab. J Clin Oncol. 2014; 32:1020-1030.

19. Robert C, Ribas A, Wolchok JD, et al. Anti-programmed- death-receptor-1 treatment with pembrolizumab in ipilimumab-refractory advanced melanoma: a randomized dose comparison cohort of a phase 1 trial. Lancet. 2014; 384:1109-1117.

20. Nguyen H, Shah K, Waguespack SG, et al. Immune checkpoint inhibitor related hypophysitis: diagnostic criteria and recovery patterns. Endocr Relat Cancer. 2021; 28:419-431.

Received January 19, 2021; Revised August 22, 2021; Accepted August 27, 2021

*Address correspondence to:

Pooja Gupta, Department of Pharmacology, Room 5004, Convergence block, All India Institute of Medical Sciences (AIIMS), New Delhi, India.

E-mail: drgupta.pooja@gmail.com

Released online in J-STAGE as advance publication August 29, 2021. 